\title{
SUPEROVULACIÓN CON PMSG APLICADA EN FASE FOLICULAR Y FASE LUTEAL EN ALPACAS
}

\author{
Carlomagno Velásquez V. ${ }^{1}$ y César Novoa M. $^{2}$
}

\section{Abstract}

Superovulatory response after PMSG and LH hormones administration during follicular phase and induced luteal phase was evaluated in alpacas. The experiment was carried out during march and April, 1996, at la Raya, IVITA Camelids Research Station, located at $4200 \mathrm{~m}$. above sea level, $15^{\circ}$ south, and $71^{\circ}$ west. For this, fifteen empty females alpacas of the Huacaya breed, ranging from 2 to 11 years of age were divided in 3 equal groups $(n=5)$ and assigned to the following treatments: $T_{1}$ (control): day 0,2 $\mathrm{ml}$ destilled water, i.m. injection; $\mathrm{T}_{2}$ (follicular phase): day 0, 1000 IU PMSG, i.m.; day 6, $1000 \mathrm{IU}$ hCG, i.m.; and $\mathrm{T}_{3}$ (induced luteal phase): day -7 , progesterone application (vaginal pessaries); day 0, 1000 IU PMSG, i.m.; day 2, remove progesterone pessaries; day $6,1000 \mathrm{IU}$ hCG, i.m. The ovarian response to the superovulation stimulation was ascertained by recording the number of corpora lutea $(C L)$ through laparotomy and by measuring progesterone levels in blood circulation by RIA. The number and size of

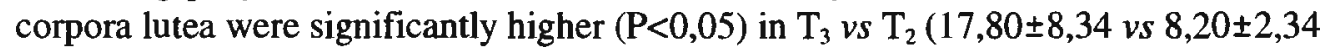
$\mathrm{CL}$, and $13,64 \pm 2,00$ vs $10,48 \pm 0,78 \mathrm{~mm}$, respectively). As regards the blood progesterone levels $(\mathrm{nmol} / 1)$, the results on day 0 were basal $\left(\mathrm{T}_{1}=0,96 \pm 0,76 ; \mathrm{T}_{2}=\right.$ $1,17 \pm 1,03$ and $\left.T_{3}=3,51 \pm 0,97\right)$. However on day 16 these levels in $T_{2}(44,27 \pm 30,17)$ and $\mathrm{T}_{3}(46,98 \pm 22,91)$ increased drastically but in $\mathrm{T}_{1}$, remained low $(1,09 \pm 0,39)$. The correlation between $\mathrm{CL}$ formed and blood progesterone levels measured on day 16 , was very low $(r=-0,078 ; P>0,05)$. We conclude that $P M S G$ followed by $h C G$ represent a good alternative for inducing superovulation in alpacas. However, from our results it would be advisable to investigate the effects of such hormones in lower dosis upon superovulation.

Key words: alpaca, superovulation, progesterone, corpus luteum.

\section{Resumen}

Se comparó la respuesta ovulatoria a la estimulación con PMSG aplicada en fase folicular versus fase luteal inducida, en alpacas. El estudio se realizó en la estación experimental del IVITA "La Raya" - Cusco ( $15^{\circ}$ latitud sur, $71^{\circ}$ longitud oeste), durante los meses de marzo - abril de 1996. Se usaron 15 alpacas Huacaya, vacías, entre 2 a 11 años de edad, que fueron divididas en tres grupos iguales, los que recibieron al azar los siguientes tratamientos: $T_{1}$ (testigo): día $0,2 \mathrm{ml}$ de agua destilada; $T_{2}$ (fase folicular): día 0, 1000 UI PMSG; día 6, 1000 UI hCG; T $_{3}$ (fase luteal inducida): día - 7, aplicación vaginal de Progesterona (CIDR); día 0, 1000 UI PMSG; día 2, retiro del dispositivo 
CIDR; día 6, 1000 UI hCG. La respuesta ovulatoria fue medida verificando el número de cuerpos lúteos (CL) mediante laparatomía y determinando los niveles de progesterona plasmática con la prueba de RIA. El número y tamaño de los cuerpos lúteos formados en $\mathrm{T}_{3}(17,8 \pm 8,3 \mathrm{CL}$ y $13,6 \pm 2,0 \mathrm{~mm})$ fueron mayores $(\mathrm{P}<0,05)$ a los registrados en $\mathrm{T}_{2}$ $(8,2 \pm 2,3 \mathrm{CL}$ y $10,5 \pm 0,8 \mathrm{~mm})$. Con respecto a los niveles de progesterona plasmática $(\mathrm{nmol} / \mathrm{L})$, el día 0 , fueron basales en $\mathrm{T}_{1}$ y $\mathrm{T}_{2}(0,96 \pm 0,76$ y $1,17 \pm 1,03 \mathrm{nmol} / \mathrm{L}$, respectivamente) y ligeramente elevadas en $T_{3}(3,51 \pm 0,97 \mathrm{nmol} / \mathrm{L})$; mientras que en el día 16 mostraron un incremento marcado tanto en $T_{2}$ como en $T_{3}(44,27 \pm 30,17$ y $46,98 \pm 22,91 \mathrm{nmol} / \mathrm{L}$, respectivamente), en cambio $\mathrm{T}_{1}$ continuó con niveles basales $(1,09 \pm 0,39)$. La correlación entre el número de cuerpos lúteos formados y los niveles de progesterona plasmática medida al finalizar el experimento (día 16) fue prácticamente inexistente $(r=-0,78 ; P>0,05)$. Se concluye, que el uso de la PMSG seguido de hCG es una alternativa para estimular la superovulación en alpacas. Sin embargo, se requiere probar dosis menores que las empleadas, particularmente en el $\mathrm{T}_{3}$ donde se observó un crecimiento ovárico excesivo con fuerte presión en la pared de la bolsa ovárica.

Palabras clave: alpaca, superovulación, progesterona, cuerpo lúteo.

\section{Introducción}

El fomento de la crianza de Camélidos Sudamericanos, particularmente de la alpaca, debe contribuir al bienestar de un sector importante de población que habita los andes altos; dichos recursos son fuente de ocupación e ingresos, de carne, fibra y de otros bienes de gran valor. Para dicho propósito se requiere, entre otros aspectos, mejorar las prácticas tecnológicas de las crianzas a fin de elevar la producción y productividad.

En esta consideración, un aspecto que merece atención es el mejoramiento genético y para ello varias instituciones están trabajando en la formación de núcleos genéticos orientados a la selección y producción de animales genéticamente superiores desde el punto de vista productivo, los mismos que son usados como reproductores en los rebaños que se desean mejorar. Sin embargo, existe una limitante, para difundir rápidamente este material genético deseado, la tasa reproductiva de los animales es muy lenta; en efecto, una hembra produce un promedio de cuatro crías durante toda su vida productiva. Esta limitación se podría superar usando la técnica de ovulación múltiple $(\mathrm{OM})$ y transferencia de embriones (TE) que harían posible que una hembra selecta pueda tener una descendencia numerosa.

En los camélidos, los estudios sobre la OM y TE son escasos y con resultados variables e inconsistentes. Los métodos superovulatorios ensayados han logrado estimular la formación de 2 a 11 cuerpos lúteos por animal, logrando recuperar sólo el 50\% de los embriones (Del campo et al., 1995). Estas respuestas son inferiores a las obtenidas en otras especies domésticas como, la vaca (Kacmarick et al., 1987); oveja (Evans et al., 1994) y la cabra (Rosnina et al., 1992). Por otro lado, de 65 transplantes de embriones realizados en camélidos se ha registrado el nacimiento de sólo 7 crías, a nivel mundial. Por lo antes expuesto, se requiere ampliar los estudios sobre este tema incluyendo procedimientos para estimular la superovulación. Este trabajo tiene como objetivo principal comparar el efecto de la PMSG aplicada en la fase folicular y en fase luteal inducida, sobre la superovulación. 


\section{Material y Métodos}

\section{Ubicación}

El estudio se realizó en la estación experimental de camélidos "La Raya", perteneciente al Instituto Veterinario de Investigaciones Tropicales y de Altura (IVITA) - Universidad Nacional Mayor de San Marcos, durante los meses de marzo y abril de 1996. Este centro experimental está ubicado en el distrito de Maranganí, provincia de Canchis, región Inca, Perú; a una altitud de 4200 metros; $15^{\circ}$ latitud sur, $71^{\circ}$ longitud oeste.

\section{Tratamientos}

Se seleccionaron 15 hembras adultas, que mostraron celo ante la presencia de macho. Estas hembras fueron divididas en tres grupos iguales $(n=5)$ y recibieron al azar los siguientes tratamientos: Testigo $\left(\mathrm{T}_{1}\right)$ : día 0 ; inyección de $2 \mathrm{ml}$ de agua destilada, vía i.m. Fase folicular $\left(\mathrm{T}_{2}\right)$ : día 0 , inyección de 1000 UI PMSG', vía i.m.; día 6: inyección de 1000 UI hCG ${ }^{2}$ vía i.m. y Fase luteal inducida $\left(\mathrm{T}_{3}\right)$ : día -7: aplicación del dispositivo $\mathrm{CIDR}^{3}$, vía vaginal; día 0 : inyección de 1000 UI PMSG, vía i.m.; día 2, retiro del dispositivo CIDR; día 6: inyección de 1000 UI de hCG, vía i.m.

\section{Mediciones realizadas}

Para evaluar la respuesta ovárica a los tratamientos se midieron los cambios en niveles de progesterona sanguínea y el número y tamaño de los cuerpos lúteos. Los

1.Gonadotropina sérica de yegua gestante ("Folligon", Laboratorio Intervet - Holanda).

2.Gonadotropina coriónica humana ("Pregnyl", Laboratorio OSS - Holanda).

3.Liberador Interno controlado de drogas, que contiene $0,33 \mathrm{~g}$ de Progesterona (EAZI BREED, Inter-Ag.- Nueva Zelandia). niveles de progesterona se midieron antes y después de la aplicación de PMSG y para tal efecto se colectaron $5 \mathrm{ml}$ de sangre de la vena yugular, los días 0,6 y 16 del período experimental.

Adicionalmente en el grupo $\mathrm{T}_{3}$, se colectó sangre, 7 días antes del día 0 (inmediatamente antes de la inserción del dispositivo CIDR). La sangre fue centrifugada durante 10 minutos a 1500 r.p.m. y el sobrenadante transvasado a frascos de vidrio estériles, los que permanecieron almacenados bajo temperatura de congelación $\left(-20^{\circ} \mathrm{C}\right)$, hasta su procesamiento.

La determinación de progesterona plasmática se realizó mediante la técnica de fase sólida de Radioinmunoensayo (RIA), siguiendo el procedimiento establecido por la Agencia Internacional de Energía Atómica (IAEA, 1990). El análisis se efectúo en el laboratorio de Reproducción animal de la Facultad de Medicina Veterinaria de la Universidad Nacional Mayor de San Marcos.

Para el registro del número y tamaño de los cuerpos lúteos se realizó una laparatomía a través de la línea media ventral, de acuerdo al procedimiento establecido por Dietz et al. (1975); con base en los estudios sobre formación, desarrollo y regresión del cuerpo lúteo realizados por Fernández-Baca et al. (1970) y Adams et al. (1990), esta intervención se realizó a los 10 días post-inyección de hCG.

\section{Análisis de los Datos}

Se utilizó un Diseño Completamente Aleatorio (DCA) con tres tratamientos y cinco repeticiones. El número de cuerpos lúteos fue analizado usando la prueba de $\mathrm{Ji}$ cuadrado $\left(\mathrm{X}^{2}\right)$. El tamaño de los cuerpos lúteos y los niveles de progesterona 
plasmática fueron analizados mediante el Análisis de Varianza (ANVA) y para la comparación entre promedios se utilizó la prueba de tukey. Para determinar el grado de asociación entre el número de cuerpos lúteos y los niveles de progesterona plasmática se utilizó el análisis de correlación (Calzada, 1983).

\section{Resulmolos y Discrion}

\section{Formación de cuerpos lúteos}

En el Cuadro 1 se muestra el número de cuerpos lúteos formados en los diferentes tratamientos.

Nótese que ningún animal del grupo $\mathrm{T}_{1}$ ovuló; sin embargo todos los animales tratados con PMSG (Fig.1) tuvieron una gran respuesta ovulatoria, pero en el grupo $\mathrm{T}_{3}(17,80 \pm 8,34$ C.L.) fue significativamente mayor $(\mathrm{P}<0,05)$ que en el grupo $\mathrm{T}_{2}$ $(8,20 \pm 2,58$ C.L.). Estos resultados, tanto en $\mathrm{T}_{2}$ como en $\mathrm{T}_{3}$, son mayores a los informados por otros estudios en la alpaca (Sumar y Franco, 1974) y en la llama (Bourke et al., 1992; Bravo et al., 1995). Una mayor respuesta superovulatoria, como consecuencia de la estimulación con PMSG en fase luteal, también ha sido descrita en la vaca (Betteridge, 1977 y Bevers et al., 1993) y en la cabra (Moore, 1974; Rosnina et al.,1992 y Córdova et al., 1992); probablemente la mayor respuesta se deba a la acción de la PMSG sobre un ovario con una población numerosa de folículos pequeños retenidos por acción de la progesterona, como ha sido descrito en vacas (Mac Millan and Vishwananth, 1994). Del total de cuerpos lúteos formados ( $\mathrm{n}=130)$ en $\mathrm{T}_{2}$ y $\mathrm{T}_{3}$, el $54,6 \%$ se localizó en el ovario derecho y el $45,4 \%$ en el ovario izquierdo, diferencia que no es significativa $(\mathrm{P}<0,05)$. Estos resultados son similares a los descritos en la llama por Aller y Alberio et al. (1996) y Bravo et al. (1995) e indican que ambos ovarios son igualmente funcionales.

En el Cuadro 2 se podrá apreciar que el diámetro de los cuerpos lúteos en $\mathrm{T}_{3}$ $(13,64 \pm 2,00 \mathrm{~mm})$ fue significativamente mayor $(\mathrm{P}<0,05)$ que en $\mathrm{T}_{2}(10,48 \pm 0,78 \mathrm{~mm})$. Esta observación también ha sido registrada en la vaca y se explica que este mayor tamaño se debe a que los cuerpos lúteos se originan de folículos preovulatorios grandes de mayor masa celular (Mac Millan and Vishwananth, 1994). Es interesante destacar que en el grupo $\mathrm{T}_{3}$ dos animales presentaron en total 8 quistes foliculares (15 a $35 \mathrm{~mm}$ de diámetro); esta anormalidad también ha

Cuadro 1. Cuerpos lúteos formados en animales testigo $\left(\mathrm{T}_{1}\right)$, en animales tratados con PMSG en fase folicular $\left(\mathrm{T}_{2}\right)$ y en fase luteal inducida $\left(\mathrm{T}_{3}\right)$.

\begin{tabular}{|c|c|c|c|c|}
\hline \multirow[t]{2}{*}{ Tratamiento } & \multirow{2}{*}{$\begin{array}{l}\text { Animales } \\
\text { (n) }\end{array}$} & \multicolumn{3}{|c|}{ Cuerpos Lúteos } \\
\hline & & Total. & Promedio & Rango \\
\hline $\mathrm{T}_{1}$ : testigo & 05 & 00 & $00^{\mathrm{a}}$ & 00 \\
\hline $\mathrm{T}_{2}$ : fase folicular (PMSG $+\mathrm{hCG}$ ) & 05 & 41 & $8,20 \pm 2,58^{b}$ & $4-11$ \\
\hline $\begin{array}{l}\mathrm{T}_{3} \text { : fase luteal inducida }\left(\mathrm{P}_{4}+\mathrm{PMSG}+\right. \\
\text { hCG })\end{array}$ & 05 & 89 & $17,80 \pm 8,34^{\mathrm{c}}$ & $5-27$ \\
\hline
\end{tabular}


Cuadro 2. Diámetro de los cuerpos lúteos ( $\mathrm{mm}$ ) en animales tratados con PMSG en fase folicular $\left(\mathrm{T}_{2}\right)$ y en fase luteal inducida $\left(\mathrm{T}_{3}\right)$.

\begin{tabular}{lccc}
\hline \multicolumn{1}{c}{ Tratamiento } & Número & Tamaño & Rango \\
& & & \\
\hline $\mathrm{T}_{2}:$ fase folicular (PMSG + hCG) & 41 & $10,48 \pm 0.78^{\mathrm{a}}$ & $9,63-11,37$ \\
$\mathrm{~T}_{3}: \begin{array}{l}\text { fase luteal inducida }\left(\mathrm{P}_{4}+\mathrm{PMSG}+\right. \\
\text { hCG) }\end{array}$ & 89 & $13,64 \pm 2,00^{\mathrm{b}}$ & $10,62-15,87$ \\
& & \\
\hline
\end{tabular}

Letras diferentes indican diferencias significativas $(\mathrm{P}<0,05)$

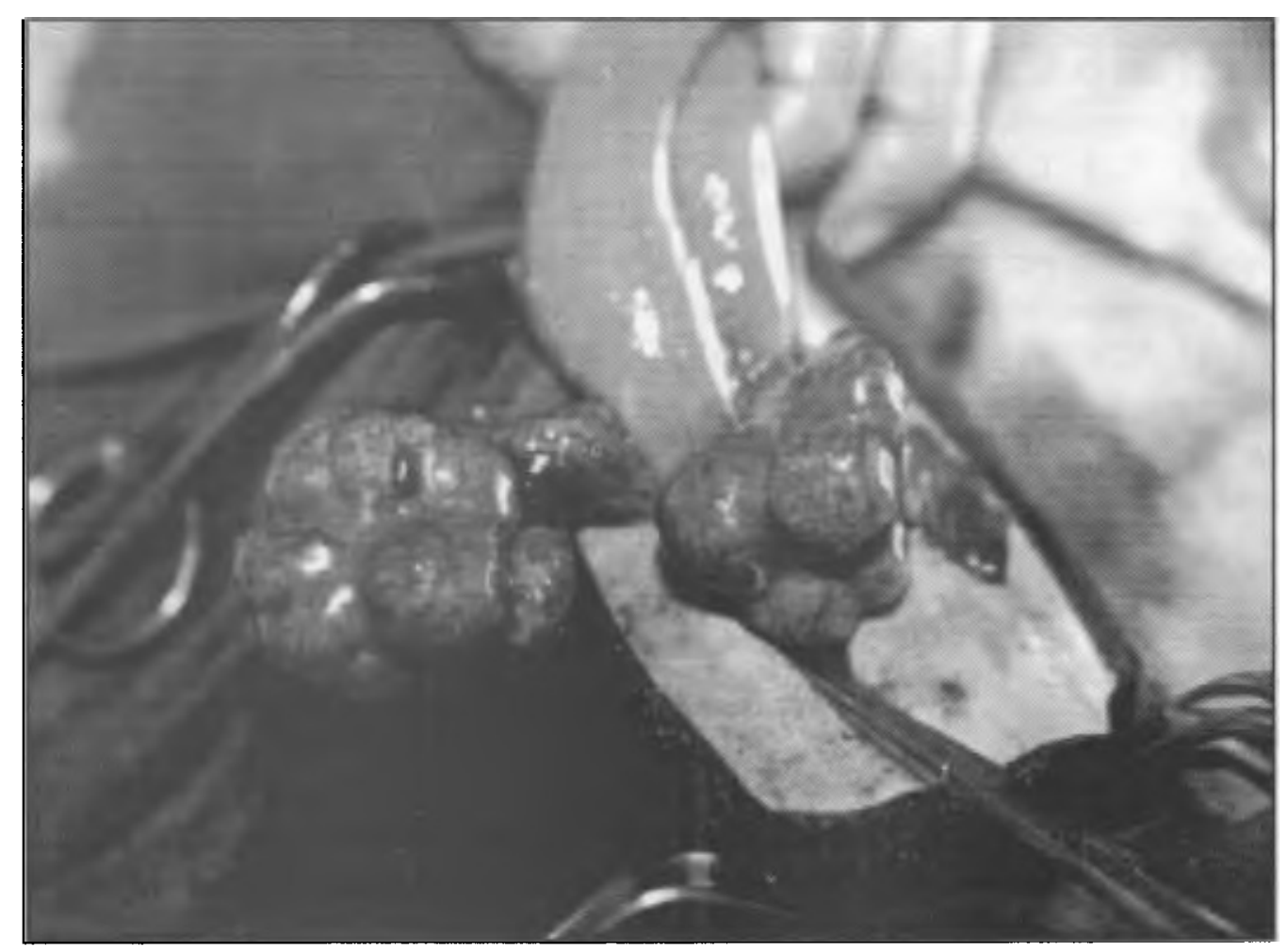

Figura 1. Ovarios de alpacas 880 superovuladas con P4 + PMSG + hCG (fase progestacional) luego de realizar una laparatomía (10 días posteriores a la aplicación de hCG). Nótese el alto número (16) de cuerpos lúteos.

sido descrita en la alpaca, por Correa et al. (1994) y en la llama, por Bravo et al. (1995). Estos últimos autores registraron un $20 \%$ de animales con quistes foliculares luego de administrar PMSG, incrementando a $90 \%$ cuando la dosis de PMSG aumentó a 2000 UI.

\section{Niveles de progesterona plasmática}

La variación en los niveles de progesterona plasmática registrada en los diferentes tratamientos, se encuentra resumida en el Cuadro 3. 
Cuadro 3. Niveles de progesterona plasmática (nmol/L) antes (dia 0 ) y después (dia 6 y 16) de la aplicación de PMSG en alpacas del grupo testigo $\left(T_{1}\right)$, en fase folicular $\left(\mathrm{T}_{2}\right)$ y fase luteal inducida $\left(\mathrm{T}_{3}\right)$.

\begin{tabular}{lccc}
\hline \multicolumn{1}{c}{ Tratamiento } & Dia 0 & Dia 6 & Dia 16 \\
\hline $\mathrm{T}_{1}:$ testigo & & & \\
$\mathrm{T}_{2}:$ fase folicular (PMSG + hCG) & $0,96 \pm 0,76^{\mathrm{a}}$ & $1,09 \pm 0,39^{\mathrm{a}}$ & $1,17 \pm 1,80^{\mathrm{a}}$ \\
$\mathrm{T}_{3}:$ fase luteal inducida ( $\mathrm{P}_{4}+\mathrm{PMSG}+$ & $1,17 \pm 5,08^{\mathrm{b}}$ & $9,30 \pm 5,08^{\mathrm{b}}$ & $44,27 \pm 30,17^{\mathrm{b}}$ \\
& $3,51 \pm 0,97^{\mathrm{c}}$ & $7,81 \pm 4,05^{\mathrm{b}}$ & $46,98 \pm 22,91^{\mathrm{b}}$ \\
& & &
\end{tabular}

Letras diferentes en las columnas indican diferencias significativas $(\mathrm{P}<0,05)$

En el dia 0 se podrá notar que los niveles de progesterona plasmática fueron basales en $T_{1} y T_{2}$, en cambio en $T_{3}$ estuvieron ligeramente incrementados. Estos resultados se encuentran dentro de lo esperado; el incremento de progesterona en el $T_{3}$ se explica por el tratamiento con progestágeno (CIDR) que se empleó para inducir la fase luteal en dicho grupo. Similares respuestas, luego de utilizar CIDR, fueron descritas en la vaca (Mac Millan and Vishwananth, 1994). Por otro lado debe destacarse que tanto en $T_{1}$ como en $T_{2}$ se registró un animal con elevación de niveles de progesterona, compatible con ovulación $(12,4$ y $7,5 \mathrm{nmol} / \mathrm{L})$. Es probable que estos animales ovularon espontáneamente como ha sido registrado en otros estudios en la alpaca (Fernández-Baca et al., 1970; Sumar 1993) y la llama (Adams et al., 1990).

Los niveles elevados de progesterona plasmática registrados el día 6 del experimento, tanto en los animales del $\mathrm{T}_{2}$ y $\mathrm{T}_{3}$, podría ser consecuencia del efecto prolongado de la PMSG; que, además de su efecto sobre el desarrollo folicular, también tiene efecto luteinizante. Resultados similares luego de aplicar PMSG fueron registrados en la vaca (Newcomb and Rowson, 1976) y en la oveja (Jabbour and Evans, 1991).

Al finalizar el experimento (día 16), los niveles elevados de progesterona plasmática registrados en los animales del $\mathrm{T}_{2}$ y $\mathrm{T}_{3}$ es consecuencia del efecto de la PMSG, que estimuló un gran desarrollo folicular múltiple, estos folículos luego de la aplicación de hCG se transformaron en muchos cuerpos lúteos. Similares observaciones, luego de aplicar PMSG, fueron reportados en la llama (Bravo et al., 1995) y en la vaca, donde la progesterona plasmática alcanzó niveles de 60 a 100 nmol/L (Spilman et al., 1973). La falta de correlación $(r=-0,78 ; \mathrm{P}<0,05)$ entre el número de cuerpos lúteos y los niveles de progesterona plasmática, registrados al finalizar el experimento, se debería a la gran variación de la respuesta ovulatoria (C.V. = 47\%). Resultados similares, luego de aplicar PMSG, fueron reportados en la llama (Bravo et al, 1995). Sin embargo, en otras especies, como en la vaca (Saumandé et al., 1985) y cabra (Córdova et al., 1992), se ha registrado una correlación positiva entre ambas variables.

\section{Conclusiones}

La aplicación de PMSG + hCG (fase 
folícular) y de $\mathrm{P}_{4}+\mathrm{PMSG}+\mathrm{hCG}$ (fase luteal inducida) resultó en una gran respuesta ovulatoria en alpacas.

La respuesta ovulatoria del tratamiento en fase luteal inducida $\left(\mathrm{T}_{3}\right)$ fue mayor que la registrada en fase folicular $\left(T_{2}\right)$, tanto en el mayor número y tamaño de los cuerpos lúteos formados.

La respuesta ovulatoria en $\mathrm{T}_{2}$ y $\mathrm{T}_{3}$ fue muy variable, no existiendo correlación entre el número de cuerpos lúteos y los niveles de progesterona plasmática, medidos 10 días después de la aplicación de hCG.

Se recomienda experimentar con menores dosis de PMSG, para evitar una excesiva estimulación del crecimiento folicular y la formación de quistes foliculares, particularmente en el $\mathrm{T}_{3}$ (fase luteal inducida).

\section{Agradecimientos}

Nuestro reconocimiento al CONCYTEC por su contribución al financiamiento del estudio y al personal profesional de la estación experimental "La Raya", por las facilidades brindadas para la ejecución de la parte experimental del estudio.

\section{Liferatura Ciada}

I. Adams, P., J. Sumar y O. Ghinter. 1990. Hemorragie ovarian follicles in llamas. Theriogenology 35:557-568.

2. Aller, J.F. y R.H. Alberio. 1996. Dinámica folicular en llamas en la época otoño invernal. Re. Arg. Prod. Anim. Vol. 16(4):319-323.

\section{Bevers, M.M., S.J. Dieleman, J.T.}

Gielen, Y.A. Wurth, B.P. Janszen, J. Van de Broeck y A.H. Willense. 1993. Yield of embryos in PMSG superovulated cows treated with antiPMSG six or 18 hours afther the peack of luteinizing hormone. Vet. Rec.132:8, 186-9.

4. Betteridge, K.J. 1977. Embryo transfer in farm animals. A review of tecniques and aplications. Agriculture Canada. Monograph 16:92 pp.

5. Bourke, A., C.L. Adam y L. Kyle. 1992. Superovulation and embryo recovery in the llama. J Reprod and fertil. Annual conference Sheffienld, 4th-6th july. Abstract 67:39.

6. Bravo, W., T. Tsutsui y L. Lasley. 1995. Dose responce to equine chorionic gonadotropin and subsequent ovulation in llamas. Small Ruminant Research 18:157-163.

7. Calzada, J. 1983. Estadística general con énfasis en muestreo. 3era edición; editorial Milagros S.A., Lima - Perú, 527 pp.

8. Córdova, S. L., K.F. Jiménez y L.J. Hernández. 1992. Superovulación con hormona folículo estimulante (FSH) o gonadotropina sérica de yegua preñada (PMSG) y anticuerpos monoclonales contra PMSG en cabras fuera de la época reproductiva. Veterinaria México 23(4):319-24.

9. Correa, J., H. Ratto y R. Gatica. 1994. Actividad estral y respuesta ovárica en alpacas y llamas tratadas con progesterona y gonadotrofinas. Arch Med Vet. (Chile); XXVI (1):59-64.

10. Del Campo, M., H. Del Campo, G. Adams y R. Mapletoft. 1995. The 
aplication of new reproductive technologies to south american camelids. Theriogenology 43:21-30.

11. Dietz, O., F. Schaetz, H. Scheleiter y R. Teuscher. 1975. Operaciones y Anestesia de los animales pequeños y grandes. 2da edición, Ed. Acribia, España 556 pp.

12. Eazi Breed CIDR. 1994. En: Anales del XXIV Seminario de la Sociedad de ovinos y vacunos de carne. Asociación Veterinaria de Nueva Zelandia. Publicación 159.

13. Evans, G., J. Brooks, W. Struthers y A.S. McNeilly. 1994. Superovulation and embryo recovery in ewes treated with gonadotrophin-releasing hormone agonist and purified follicle-stimulating hormone. Reprod. Fertil-Dev; 6(2): 24752.

14. Fernández-Baca, S., W. Hansel y $C$. Novoa. 1970. Corpus luteum function in the alpaca. Biol. Reprod. 3(2):252261.

15. I.A.E.A. 1990. Livestock reproduction in Latin america. Proceding of the FAO/ IAEA. División of nuclear Techniques in Food Agriculture. Bogotá-Colombia, 19 - 13 sept 1988. International Atomic Energy Agency. Vienna 446 p.

16. Jabbours, H. N. y G. Evans. 1991. Ovarian and endocrine responses of Merino ewes to treatment with PMSG and for FSH. Animal Reproduction Sciencie 26:93-106.

17. Kacmarick, J., P. Gamcik, J. Posivack, D. David, P. Hruskova y E. Piesova. 1987. Superovulation after administration of PMSG in cows of various breeds. Vet. Med. (Praha)32:10, 577-86.
18. Mc Millan, W. y R. Vishwanath. 1994. Sincronización de celos e inseminación artifical en vacunos de carne. Anales del XIV seminario de la sociedad de ovinos $y$ vacunos de carne. Asociación Veterinaria de Nueva Zelandia. Publicación 159.

19. Moore, N. W. 1974. Múltiple ovulation and ovum transfer in the goat. Proc australian Soc. Anim. Prod. 10:246-249.

20. Newcomb, R. y J.E.A. Rowsom. 1976. Múltiple ovulation egg transplantion: Towards twining. In principles of cattle production. Ed. Swan and W. H. Broster. Proc. 23rd Easter School in Agricultural Sciencie, University of Nottinghain. Butterworths, London 59-83.

21. Rosnina, Y., M.R. Jainudeen y M. Nihayah. 1992. Superovulation and egg recovery in goats in the tropics. Vet. Rec. 130(5):97-9.

22. Saumande, J., D. Tambousa y D. Chupin. 1985. Changes in milk and plasma concentrations of progesterone in cows after treatment to induce superovulation and their relationships with number of ovulations and embryos collected. Theriogenology. 23:719.

23. Spilman, C. H., G.E. Seidel (jr), L.L. Larson, G.R. Vukman y R.H. Foote. 1973. Progesterone 20 betahidroxipregn-4-en-3one, - and luteinizyng hormone levels in superovulated prepuberal and postpuberal cattle. Biol. Reprod. 9:116124.

24. Sumar, J. y E. Franco. 1974. Ensayo de Transferencia de Embriones en Camélidos Sudamericanos. En: Informe Final (IVITA). Univ. N.M.S. Marcos. Lima, Perú. 
25. Sumar, J. 1993. Efectos de los estímulos de inducción en la ovulación de alpacas y llamas. Rev. Inv Pec.IVITA (Perú) 6(1):17-21. 